\title{
Antiretroviral treatment use and HIV-RNA suppression rates for 877 European patients in the etravirine expanded access programme
}

\author{
E Florence*1, S De Wit ${ }^{2}$, A Castagna ${ }^{3}$, E Ribera ${ }^{4}$, AM Hill5, R Ryan ${ }^{6}$, \\ H Vanaken ${ }^{7}, \mathrm{~S}_{\text {Marks }}{ }^{2}$ and Y van Delft ${ }^{8}$
}

Address: ${ }^{1}$ Prince Leopold Institute of Tropical Medicine, Antwerp, Belgium, ${ }^{2}$ St Pierre University Hospital, Brussels, Belgium, ${ }^{3}$ RCCS, Fondazione San Raffaele del Monte, Milan, Italy, ${ }^{4}$ Hopital Vall d'Hebron, Barcelona, Spain, ${ }^{5}$ Liverpool University and Tibotec BVBA, Liverpool, UK, ${ }^{6}$ Tibotec R \& D, Yardley, USA, ${ }^{7}$ Tibotec BVBA, Mechelen, Belgium and ${ }^{8}$ Janssen-Cilag B.V., Tilburg, Netherlands

* Corresponding author

from Ninth International Congress on Drug Therapy in HIV Infection

Glasgow, UK. 9-13 November 2008

Published: 10 November 2008

Journal of the International AIDS Society 2008, I I (SuppI I):P36 doi: I0.I I86/I758-2652-II-SI-P36

This abstract is available from: http://www.jiasociety.org/content/II/SI/P36

(c) 2008 Florence et al; licensee BioMed Central Ltd.

\section{Background}

The next generation NNRTI etravirine (ETR, TMC125) has shown strong and durable efficacy in the DUET trials in combination with a background regimen of darunavir/r, NRTIs and optional enfuvirtide. The expanded access programme included a wider range of background regimens (BR).

\section{Methods}

The TMC125-C214 trial (etravirine expanded access programme) included patients with triple class experience (NRTI, PI, NNRTI) and who were unable to use currently approved NNRTIs owing to either intolerance or drug resistance. Patients were recruited from ten countries in Europe and received etravirine $200 \mathrm{mg}$ BID with a range of background antiretrovirals.

\section{Summary of results}

By $31^{\text {st }}$ May 2008, there were 877 European patients with data available: $21 \%$ were female, $87 \%$ were Caucasian, with a mean age of 46 years. The baseline mean CD4 count was 278 cells/uL (range 0-1647) with baseline mean HIV-RNA 5248 copies/mL (range $40-3,263,277$ ). $667 / 882(76 \%)$ patients used at least one PI, of which 606 (91\%) used DRV/r. 251/882 (28.5\%) patients did not use NRTIs in the background regimen. Of the 631 patients using NRTIs, the most common were tenofovir (69\%),
3TC or FTC (91\%), ZDV (20\%) and ABC (18\%). Other antiretrovirals used included raltegravir (54\%) and maraviroc $(14 \%)$. The percentage of patients with HIV-RNA $<50$ copies/mL was $13 \%$ at week $4,48 \%$ at week 12 and $67 \%$ at week 24 (observed data analysis). CD4 counts rose by mean 75 cells/uL at week 12 and by 89 cells/uL at week 24. There were 131 serious adverse events (SAE's) recorded, of which 117 (89\%) were judged to be not related to etravirine or doubtful causality. There were 14 SAE's judged to be at least possibly related to etravirine. Of these, there were five $(0.6 \%)$ cases of rash (ETR permanently stopped in 4/5 cases), one elevation of ALT levels $(0.1 \%)$ (ETR dose not changed) and one case of cholestatic acute hepatitis $(0.1 \%)$ (ETR dose not changed).

\section{Conclusion}

The use of etravirine in the expanded access programme included a wide range of antiretrovirals in the background regimen, with a high percentage of patients showing HIVRNA suppression below 50 copies/mL by weeks 12-24. Serious adverse events were mainly unrelated to ETR. 